\title{
Next generational fuji IX-a proposed universal dental material-but not yet 'set in cement'
}

\author{
John A. Loudon \\ Correspondence: jon.uk1515@gmail.com

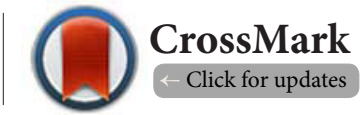

Wetherill Park Medical Centre, Suite 101, Stockland Mall, Polding Street, Wetherill Park, Sydney, NSW, 2164 Australia.

\begin{abstract}
In this article a summary from the literature is presented for the use of next generational conventional glass ionomer cement (Fuji IXGP) for a plethora of dental situations. Applications include not only restorative but also cementation and core buildup scenarios. Fuji IXGP fluoride release, radio-opacity and reasonable aesthetics offer-up advantages in field and Special Needs dentistry as well as in the clinic for caries susceptible individuals. The adaptability of such a material thus ranks it highly for virtually universal use in clinical practice and encapsulation frees the operator from variability in hand-mixing. Personal experience in many clinical situations with this material is recounted. Downsides in terms of its physical properties are outlined and means and ways to aid addressing these aspects are portrayed. Further, along these lines, it is noted that the so-called 'ideal' biocompatible restorative dental material is not available as yet and the field is undergoing constant re-evaluation and change. Clinicians should be flexible to incorporate novel approaches into their dentistry based on individual experience. Ultimately, sound, evidence-based randomized controlled, blinded, clinical trials determine the best direction to pursue yet oftentimes anecdotal findings may initiate and inspire such trials. Clinicians are urged to be pro-active in implementing research strategies into their daily routine with the concept in mind that the largest laboratory of all is, in the end, the clinic itself.
\end{abstract}

Keywords: Atraumatic restorative technique, compressive bond strength, crown cementation, dental core, dental post, dental restorative, dental core, dentinal bonding, enamel bonding, ferrule effect, Fuji IXGP, Glass ionomer cement, luting cement, root caries, shear bond strength, tensile bond strength

\section{Introduction}

There are few areas more confusing in clinical dentistry than adhesive/restorative technologies. In this article, the reader's attention is drawn towards the overall utility of next generational Fuji IXGP glass ionomer cement within the FujilX restorative class. This material may be supported by evidence for its practical use in dentistry-from luting posts and crowns as well as in core build-up and adult/paediatric restorative situations.

Conventional glass ionomer cements (GIC) were introduced into the profession in 1971 by Wilson and Kent [1], presenting as a chemical base: [ion-leachable calcium-aluminium-fluorosilicate] glass powder-combined with a polyacrylic acid. Benefits of use included adhesion to moist tooth structure with low marginal microleakage, bonding to base metals, fluoride release, low solubility in the mouth and a coefficient of thermal expansion matching that of tooth structure. The organic [ $\left.\mathrm{COO}^{-}\right]$groups of the $\mathrm{GIC}$ bind to enamel $\mathrm{Ca}^{2+}$ ions thereby resulting in a noninvasive, superficial bonding-a central property of the material.

An analysis has been very recently presented regarding ion exchange between conventional glass-ionomer restorative material-FujilX-and tooth [2]. This study is instructive in that for both enamel and dentine adjacent to glass ionomer cement restoration strontium, silica and alumina ions diffused from the restorative into the tooth substance. Importantly, fluorine showed a dramatic presence within the surrounding tooth after four years in vivo. Calcium exchange into the restorative material was also observed [2]. Ions such as strontium, alumina and fluorine migrating from the glass ionomer restorative into the surrounding dental structures may be presumed to possess a bactericidal/anti-cariogenic influence and may also enhance remineralization. Calcium ion exchange into the restorative may, in turn, aid in transforming the material into a more robust, enamel-like material [2]. The fact that these ionic movements occur actively in vivo over time is of significant clinical import and speaks highly in itself for the usefulness of glass ionomers in dentistry.

Therefore with such a broad span of valuable properties one may consider that this material may form the basis for a universal-type dental application. The author has used this new generational glass ionomer-viz: Fuji IXGP (auto-cured, encapsulated, fast-setting FujilX). on many occasions to some benefit in the dental clinic in a wide variety of clinical scenarios. The Fuji IXGP Extra and Fast set were used interchangeably by the author in the clinic and form the underlying basis for this presentation.

Overall, the purpose of this article is to present a balanced 
John A. Loudon, Oral Biology and Dentistry 2014,

discussion of the worth of this next generational glass ionomer for a wide variety of situations encountered by the dental clinician. In particular, an objective discussion of inherent downsides in each situational context is a central aim. Importantly, means to address such challenges to its use are portrayed along with scope for future developments to further enhance the material's use. It is aimed to inspire clinicians to derive anecdotal evidence in their practise of dentistry in order to drive forward evidence-based lines of enquiry that ultimately shape future improvements in dental materials science. The ultimate goal of one material, all purposes is idealistic, yet worthy of pursuit and may one day be attainable-a philosophy I follow in this article.

\section{Fuji IXGP-restorative material and cement}

It is important to note that in dental materials science numerous physical properties must be taken into consideration in order to be able to fulfill the varied and rather demanding constraints of the intraoral environment. In dentistry, there are many different situations that place demands on physical properties. Indeed, one may select a material for various purposes, for example, as cement or as core build-up material or as a restorative. Restorative materials require high compressive and tensile strengths in order to withstand the rigors of the environment imposed on them. Forces are not only seen in simple tension and compression but also in terms of shear and torsion (Figure 1). Survival of a restoration requires it

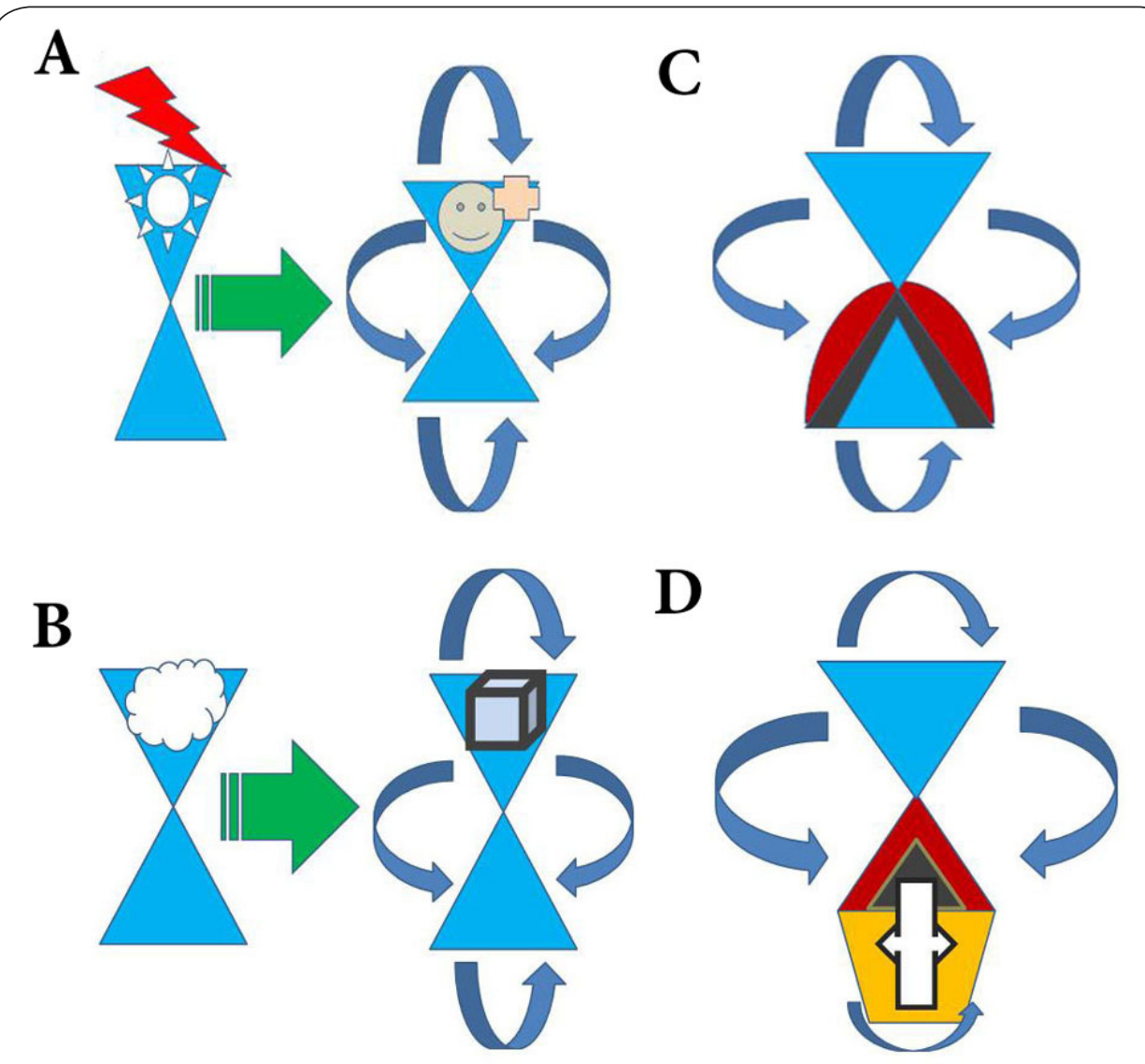

Figure 1. Fuji IXGP-an 'all-purpose' dental material.

(A). Use of FujilX to restore teeth damged by carious attack-ledermix combined with GIC may offer-up obtundent effects in addition to providing coronal restoration to teeth presenting with pulpitis.

(B). Use of FujilX as a liner or base under a dental restoration to replace cariously involved tooth structure-insulation and sealing properties become invaluable in this context-black outlined box represents restoration with liner (dark shade).

(C). Use of FujilX as a crown luting agent-dark shaded under crown.

(D). Use of FujilX as a core build-up and post luting agent-'all in one'. Dark shade represents FujilX as core and post lute.

Arrow indicates forces acting on the tooth-restorative system. ViZ: torsion shear, tensile, compressive. Triangles represent teeth-occluding pairs, upper and lower. 
to withstand such a harsh environment. The ability of the material to demonstrate packable or condensable properties is also very important-allowing manipulation of the material by the clinician to be much simpler. Fuji IXGP possesses that particular property and therefore is readily well handled in clinical scenarios. Further, restorations need to show ideally an adherence to underlying tooth structure not just for retentive features but also for minimizing leakage and subsequent marginal carious breakdown.

For dental cements, their properties are equally manifold. They are required to bond securely to tooth structure as well as have favourable engineering properties that enable them to be durable and retain the restoration they support faithfully. Cements may be called upon too to bond to radicular dentine to support posts. The bonding nature of coronal vs radicular dentine may be different hence complicating and making for a greater challenge. A material that is useful too as a liner or base to insulate the pulp of the tooth against chemical and physical challenges is required by clinicians. This material must adhere well to underlying tooth and have sufficient physical strength matching that of a general restorative.

Clearly any material that would be universally used by the dental clinician would have to 'measure up' suitably against all of the above. In the author's experience the material, Fuji IXGP, fulfills many of these criteria making for a very adaptable, 'all-rounder' conventional glass ionomer (Discussion).

In terms of precise physical properties, the compressive and diametric tensile strengths of glass ionomer are relatively acceptable-being $\sim 150 \mathrm{MPa}$ and $\sim 12 \mathrm{MPa}$ at $24 \mathrm{hr}$, respectively [3]. Despite the observation that composite and amalgam have higher values for these physico-mechanical properties, conventional glass ionomer has been shown to compare well in the clinic in terms of restoration survival [4]. This suggests from observational analysis that a minimum strength capability is required and, once over this threshold, then acceptance may be found for dental use (Discussion).

Next generational, high viscosity materials such as Fuji IXGP and Fuji IXGPExtra are available in encapsulated form and have been used extensively by the author in practice. Fuji IXGPExtra demonstrates higher fluoride release and improved aesthetic properties. A set time of $2^{1 / 2} \mathrm{~min}$ and early set strength make these new generation materials ideal for Special Needs dentistry as well. By Vickers testing, Fuji IXGP fast set had improved hardness compared to regular Fuji IX and particle sizes are lower for fast set being at $\sim 7$ micron comparing to regular set at $\sim 13$ micron [5]. The particle size value for Fuji IXGP fast set would thus appear more suited for crown cementation - as low and even particle size distribution is a necessary feature for luting cements.

Microshear bond strengths of Fuji IXGP fast set are $\sim 5-7 \mathrm{MPa}$ measured over pulpal horn areas and these strengths are not affected by conditioning dentine [6]. Dentine permeability and surface wetness notably affects resin-modified cement adhesion and also pulpal pressure and surface treatments can modify the adhesion of resin-based materials possibly leading to post-operative leakage and sensitivity [6]. Fuji IXGP fast set appears relatively indifferent to these quantities and may be said to be less technique sensitive [6]. Obviously these above factors have relevance both in cementation and restoration with Fuji IXGP - carrying certain advantages in both respects. Undoubtedly too, the fluoride release capability of glass ionomer certainly makes it attractive for restorative purposes [7].

Microtensile bond strength of Fuji IXGP fast set averages $\sim 12 \mathrm{MPa}$ to coronal dentine [8]. The resultant bond is therefore not weak and matches well the microtensile bond strength of composite and resin-modified glass ionomer cement [9]. In a well cited study, various properties of luting cements have been carefully examined [10]. Elastic moduli (stiffness) of the glass ionomers increase over time after placement. Under mechanical pressure materials can undergo plastic flow and/ or elastic deformation. Thus brittle materials with high moduli of elasticity are needed to resist marginal breakdown from plastic flow and/or microfracture and flexing. Ideally, the cement ought to have an elastic modulus between that of the tooth and the restoration in order to decrease interfacial stress and resultant physical flexural strain. An important key point here is that dislodgement is in fact lesscatastrophic than flexural marginal opening and leakage which only seeks to allow bacteria ingress with resultant caries and pulpal disease. Thus as a property, strength may be sacrificed against elastic modulus. Glass ionomers have relatively high elastic moduli, just above that of dentine which is considered ideal as this favourably distributes forces at the dentine-cement interface. Since glass ionomers are brittle they fail with little demonstrable plastic deformation with other cements showing flow before failure. Additionally, compressive strengths of glass ionomer cements compare well with other cements. In summary, microleakage is related to mechanical as well as adhesive properties and materials such as Fuji IXGP by brittle failure avoid longer term marginal flow and leakage which is detrimental either as a lute cement or restorative material.

One point however is that as Fuji IXGP is a viscous material it needs higher seating force to be used in order to achieve adequate marginal seal (Personal Perspective). The need for this added seating force has already been documented to be directly related to marginal seal with glass ionomer cement [11] (Discussion).

Glass ionomer as a cement for glass fibre posts has been shown to have acceptable tensile strength [12]. In addition, where application of adhesive technique is challenging then ionomer cements are of benefit as they are relatively less technique sensitive as has already been mentioned above. Post luting with a core build-up material has been reported to significantly increase bond strength with fibre posts to root dentine [13]. This not only simplifies the protocol but is consistent with the use of such core materials as Fuji IXGP for luting posts. Use of a combined composite core and lute 
John A. Loudon, Oral Biology and Dentistry 2014,

cement for glass fibre posts resulted in a push-out bond strength of $10+/-5 \mathrm{MPa}$ [14]. As this figure is within the range for that reported for Fuji IXGP fast set bonding to dentin [6], it can be concluded that Fuji IXGP may well be considered a suitable 'all rounder' for post lute and core. Further studies aimed at distinguishing in particular the bonding of Fuji IXGP fast set to radicular vs coronal dentine are needed to clearly further define the post-retaining strengths of this $\mathrm{GIC}$-although, as summarized above, this appears certainly likely to be favourable (Discussion).

\section{Personal perspective}

In the author's experience, Fuji IXGP performs well under a wide variety of circumstances. Firstly, as a base or liner under composite restorations near to the pulpal tissues it prevents sensitivity. Direct pulpal use is not recommended. The author uses ledermix cement containing an anti-inflammatory and anti-microbial agent for direct pulpal use where mild pulpal symptoms present under extensively decayed tooth structure. In such instances, Fuji IXGP may be used to provide for a durable 'on probation' restoration. Patients are duly informed of the situation and longer term provision of a composite surface filling may be done for added strength and aesthetics when the status and reactivity of the pulp has revealed itself over time. In fact, when teeth present with symptoms under previously laid composite restorations, the author finds it an appropriate strategy to remove the composite and dress near to the pulp in the deeper part of the old preparation with ledermix cement or a thin layer of ledermix paste. At that stage a durable coverage with Fuji IXGP is performed. Generally, the tooth is found to 'settle' within a week or two if initially found to be cold sensitive or even hot sensitive or mildly tender to touch. In order to 'fine tune', a composite restoration on top of the Fuji IXGP layer may be then placed. Pre-conditioning the tooth is not done and this simplifies the protocol and places less insult onto the pulp.

In brief, the adhesive quality of Fuji IXGP, its fluoride release, ease of placement and adequate physical properties (Discussion) lends this material for short to longer term restoration of teeth which present in a variety of circumstances with early or mild reversible pulpitis. For vital pulps not demonstrating symptoms the author recommends use of calcium hydroxide hard setting deep liner ifvery close or on the pulp followed by 'sandwiching' Fuji IXGP as a base then etching this material for bonding composite on top as a surfacing layer.

In terms of use as cement, the author has found Fuji IXGP worthwhile. Although somewhat viscous, the material may be used for cementing crowns and bridges. The caveat here is that one has to work in an organized fashion. No conditioning of the tooth is done. The tooth must be isolated and a dry field obtained as usual. The assistant mixes the encapsulated material with the dentist then taking the application syringe and placing approximately one half of the capsule into the crown fitting surface with crown seating and maintenance of a constant force on the unit during the initial $30-60$ s set period. Once initial set is reached, excess cement is easily removed and fractures readily away. Contact points are flossed. Margins are recommended to be scaled clean with an ultrasonic device after a further 10 min wait.

Another application for crown preparations is where the pulp has developed sensitivity under the preparation. This may manifest itself during the period of time temporary restorations are in place. This is not an uncommon finding in that teeth prepared for crowns become sensitive and develop pulpitis. At times, the pulp insult may be significant enough to yield an irreversible pulp damage response. Nevertheless, if sensitivity develops it may be possible to place a thin layer of ledermix paste or cement over a small portion of the preparation, usually $\sim<10-15 \%$ tooth surface area, and then cement over this the crown with Fuji IXGP as the lute agent. The ledermix layer shall displace the lute cement over the surface that is covered into the die spacer layer used to create the cementation 'gap' between crown and tooth. Thus the obtunding agent, viz: ledermix, shall simply occupy the die-spacer layer created in the laboratory that allows lute cement to normally occupy. The Fuji IXGP shall occupy the die-spacer layer around this on seating the crown and therefore seal around the area of the tooth treated with ledermix. Attention must be taken not to take the ledermix to the marginal area however, as this could compromise the cement seal. Provided no more than $\sim 15 \%$ of the tooth surface areais coated with ledermix agent, the strength of cementation appears not to be significantly compromised. The underlying tooth may then benefit from a 'medicinal jacket' placed over it at cementation that shall aid in settling any adverse pulp reaction to the preparation. It will not enable complete recovery of severely irritated pulps secondary to preparation, and these may require, in time, root canal therapy. Fortunately this outcome is uncommon at a rate of $\sim 6 \%$ [15].

In addition to standard seating of crowns and bridges Fuji IXGP may have additional uses. When a core is built-up in readiness for a crown impression and temporary placed (the fixed prosthodontic work being made in the laboratory) if, upon removal of the temporary, a portion of the core dislodges, Fuji IXGP may prove most useful. The amount of core that may fracture oftentimes only represents a relatively small percentage of the overall structure, that is, 10-15\%. It thus remains practical and feasible to use the lute material concurrently as a core reparative material. With this procedure, simply place an excess of Fuji IXGP into the crown fitting surface and seat as above and finish. The author has found that there is good retention on follow-up therefore avoiding a remake of the core and re-impression and new crown/bridge, which may prove to be time consuming and costly. In this instance, the ready adaptability of Fuji IXGP becomes evident.

Cementing dental posts and use of post lute material simultaneously as a core material provides for an 'all-in- 
one' type of approach which is very convenient. The author generally selects glass fibre posts that are parallel sided and prepared to a depth of $\sim 3 / 4$ root length. The apical $1 / 4$ root canal material ought to be assessed for adequate seal and healthy apex - as determined via intraoral radiography. The canal is cleaned of debris and dried and the post is coated liberally with Fuji IXGP, working efficiently with one's assistant as noted above for the crown lute protocol. The post is then firmly seated to depth and held till initial set and excess is simply removed. Nil prior chemical treatment nor conditioning of the post preparation is performed with Fuji IXGP use for post cementation. This again simplifies the protocol overall. Thecore is then built-up with a separate application of Fuji IXGP. After a wait period of $\sim 10 \mathrm{~min}$ the core is shaped and prepared per clinical need. Attention to occlusion is important to avoid excessive compressive/shear or torsional force on the restored post/core structure. Provision of a ferrule, if possible, is recommended. The core may also be supported by selfthreading pins into the root face to stabilize and give added retention and as an anti-rotation feature. The post depth should be greater than the height of the restoration above the root face. The particular clinician may wish to examine the survival of a temporary fixture in complex multiunit cases where support needs to be assessed prior to completion of final bridgeworks.

Finally, the author has also found it of value to repair margins of crowns that have become exposed over time due to gingival recession. This is not an uncommon malady and leads to an unattractive dark-appearing line at the margins of porcelain-fused to metal crowns which interferes significantly with anterior cosmetics. Rather than total removal of crown or perhaps bridgework, it proves convenient to prepare a slot within the labial marginal area, removing metal/porcelain and some dentinal structure. Fuji IXGP acts as an excellent opaque, or 'blocking agent' and provides a fluoride releasing dentinal-bonding seal when placed as a liner into the created slot. The area may then in turn be surfaced with shade-matching composite to the porcelain used for the unit-this adds to the cosmetics. Fuji IXGP may also be used to seal peripherally for crowns that demonstrate some degree of marginal microleakage. This may manifest as cold sensitive when drinking cold drinks or eating cold foods. Rather than replace the crown in toto, in order to aid obtaining an improved marginal seal, a slot preparation is made around the margin that is suspected of developing leakage and then sealed with Fuji IXGP. Dentinal bonding along with fluoride release may be presumed to aid in reducing marginal leakage and assist in alleviating symptoms. The patient is duly carefully cautioned to maintain strict oral hygiene around the sealed margins. The literature speaks of cosmetic repair of crown margins in composite $[16,17]$ which saves in terms of costs and convenience and yields a satisfactory end cosmetic result. A circumferential, shoulder-type means of preparation located within the enamel may be performed as the literature outlines.
A bevel can be then introduced into the cervical enamel and opposing porcelain to enable added space for a thin layer of composite to colour blend. Microleakage analysis and scanning electron microscope studies of the marginal area showed that the design used produces good marginal seal and contour supragingivally. The present author finds that Fuji IXGP serves as an alternative material that has multiple benefits in this context, such as dentinal bonding and fluoride release-important for maintaining marginal integrity against subsequent risk of plaque-related demineralization. Again, the patient must be educated thoroughly as to marginal plaque control and added aids such as mouthrinses are advised. An outline of a number of the uses of Fuji IXGP is presented (Figure 1A-1D).

\section{Discussion}

Glass ionomers have significant advantages over many dental materials in terms of the ability to release fluoride and their resultant remineralising capabilities $[\mathbf{1 8 , 1 9 ]}$. Specifically, in vitro remineralization of incipient carious lesions has been examined with Fuji IXGP, Vitremer, Ketac-Molar, or Z250 used as restorative materials [20]. Notably, there was a significant decrease in the size of caries lesions adjacent to cavities restored with glass ionomers, viz: Fuji IXGP, Vitremer, Ketac-Molar. This indicated that glass ionomers had significantly greater remineralization effects on adjacent carious lesions than the non-fluoridated composite resin with the three glass ionomers performing equally well. Encapsulated Fuji IXGP demonstrates satisfactory release of fluoride ions into tooth structure when used via ART - atraumatic restorative technique [21]. It was concluded that the pattern of penetration of caries resolving fluoride into dentine was consistent with remineralization. Thissignificantly supports the use of high strength glass ionomer cement, viz: Fuji IXGP, for restorative practice.

An important consideration oftentimes involves managing patients who present with challenging carious lesions secondary to either direct pathologies leading to xerostomia (dry mouth) or iatrogenic causes such as radiation-induced salivary gland damage. Fujil XGP used as a high strength fluoride releasing viscous glass ionomer cement has shown to be particularly advantageous in the management of secondary caries in such circumstances [22]. Interestingly, despite the fact that $\sim 12 \%$ of Fuji IXGP restorations were lost over a period of two years there were nil instances of recurrent caries. The retained glass ionomer restorations demonstrated a certain degree of marginal deterioration - yet this was not deemed to be serious to the extent of requiring repair/replacement of the restoration in question. Surface erosion was not a matter for concern either. In summary, in such 'high risk' radiation induced caries patients, placement of viscous glass ionomers such as Fuji IXGP appeared to be suitable and prevented secondary caries despite the fact that restorations were in fact lost in $\sim 1 / 8^{\text {th }}$ of cases.

A further important consideration pertains to bond stren- 
John A. Loudon, Oral Biology and Dentistry 2014,

gths of the material to underlying tooth structure. This is important not just for the obvious situation involving retention but also in terms of marginal breakdown and leakage. This may lead to bacterial ingress causing recurrent carious attack with associated pulp irritation/pathology. As already indicated above, the microtensile bond strength of Fuji IXGP averages approximately $12 \mathrm{MPa}$ to coronal dentine [8]. The resultant bond is therefore not weak. Strong and significant bonding of glass ionomers to underlying tooth structure is due to chemical bonding interactions with the mineral phase of underlying tooth [23] aided by the evident ion-exchanges [2]. It ought to be noted that reactive, sclerotic dentine under carious lesions has a different mineral composition as compared to non-carious, non-deminerlized dentine substrate. This may have relevance in terms of glass ionomer bonding. Encapsulated glass ionomer wasexamined along with other glass ionomers (hand mixed). Teeth were conditioned with $10 \%$ polyacrylic acid and shear bond strengths were examined. The encapsulated glass ionomers showed shear bond strength to sound dentine that was similar to that of sclerotic dentine. These data support the use of Fuji IXGP (encapsulated) as being suited to enable bonding to underlying sclerotic dentine as a cement or liner or indeed as a restorative.

Conditioning underlying dentinal substrate is oftentimes a clinically suggested technique. Nonetheless, evidence suggests that in respect to Fuji IXGP there is little to be gained from this procedure [24]. In that particular investigation, various glass ionomers, including Fuji IXGP, were assessed for microtensile bond strength to underlying dentine. Four different conditioners were employed in the testing. Averaged microtensile bond strength of non-conditioned specimens did not vary significantly from conditioned specimens. Cohesive failure within the glass ionomer itself was the principal mode of failure. As suggested earlier, Fuji IXGP microshear bond strengths are not affected by dentine conditioning [6]. This result is consistent with the microtensile bond strength data [24].

Another factor in bonding or adhesion of glass ionomer to underlying tooth is that of salivary contamination during placement [25]. High viscosity Fuji IXGP was assessed as to the potential effects that salivary contamination may have on the shear bond strength to conditioned dentin. Also, an assessment of cleaning the contaminated field prior to bonding was performed. Fuji IXGP performed better than Ketac-Molar in terms of shear bond strengths to dentine. Failure was mostly cohesive for Fuji IXGP and adhesive/cohesive for Ketac-Molar consistent with the superior bonding strength of Fuji IXGP. It was summarized that salivary contamination did not affect average shear bond strength of Fuji IXGP and Ketac-Molar to conditioned dentine. To what degree conditioning provides a protection from salivary contamination and ensuing bonding strength remains to be further examined for Fuji IXGP, although from the above discussion, it is feasible to surmise that conditioning may not influence the bonding reaction to a major degree.

Not with standing the above, significant concerns have been raised regarding bond strength to tooth structure of Fuji IXGP. For example, it has already been demonstrated that Fuji IXGP performed poorly against a resin modified glass ionomer cement and a compomer in regards tensile bond strength and microleakage [26]. Further, an investigation has examined the shear bond strength to enamel of rest seats made with Fuji IXGP vs resin modified glass ionomer and composite under various loading conditions [27]. It was found that Fuji IXGP resulted in the lowest shear bond strength fatigue limit $(2.33 \mathrm{MPa})$. To counter the situation in regard to poor performance in respect to bond strengths various strategies have been applied. Heating Fuji IXGP to produce a 'command set' demonstrated significantly improved shear bond strength to enamel [28]. Further, microtensile bond strength of high-viscosity GIC was significantly enhanced by ultrasonic excitation [29].

To aim in improving microshear bond strengths with Fuji IXGP, various bonding agents and protocols were assessed. It was found that self-etch and bond produced a stronger microshear interaction with Fuji IXGP compared to etch, rinse and bond [30]. This has relevance in terms of bonding to other restorative materials such as composite when using Fuji IXGP as a liner or even for use to enhance direct Fuji IXGP interaction with tooth substrate. Cohesive failure of the Fuji IXGP was a limiting feature and in itself requires to be addressed through improvements geared to increase intrinsic material strength (see below). Along such lines, a simple way to develop enhanced GIC properties is to allow time for $\mathrm{GIC}$ maturation [31]. In this respect, shear-bond and flexural strengths increased significantly after a day. It was suggested that the operator merely allow time before polishing to avoid material disturbance and marginal gap formation.

Running in parallel to bond strength is the important clinical facet of microleakage. This factor in particular may be responsible for restorative failure and patient symptoms. For Fuji IXGP, microleakage is a relevant concern [32]. It is nonetheless promising to note that, similar to the above with command-set via heating Fujl IXGP improving shear bond strength to enamel [28], a heat treatment during setting may also tend to improve microleakage score with this material [33]. Further to this, it is reassuring to appreciate that the nature of the mixing process itself is influential on the degree of clinical microleakage observed [34]. As such, encapsulated Fuji IXGP with mechanical mixing appeared to reduce significantly marginal microleakage in demanding restorative work such as class II situations as compared to bottled components-manual mixing.

Having said this, it ought to be also appreciated that other studies have indicated that Fuji IXGP, on challenging with exacting dye-penetration analysis, appears to perform in satisfactory terms in regards microleakage [35]. Another reassuring investigation indicated that Fuji IXGP was seen to 
behave much like composite and resin modified glass ionomer in regards microleakage [36].

Overall speaking, the context that drives microleakage may well go beyond consideration of a particular restorative/ cement, but rather the nature of the underlying substratedentine. The production of a suitable 'hybridised layer' relates significantly to microleakage effects [37]. In this regard, the 'take home message' would be to reliably inhibit microleakage in restorative dentistry by preparing bonded (infiltrated) demineralised dentine-the 'hybridised dentine' concept [37].

In terms of use as restorative material, it ought to be noted that intrinsic strength of the restoration itself is an ultimate test of how long the actual filling shall last. As indicated above [5], by Vickers test, Fuji IXGP had improved hardness compared to regular FujilX. Particle sizes are lower for the former being at average $\sim 7$ micron comparing to regular set at $\sim 13$ micron [5]. Fuji IXGP would thus appear quite suitable too for crown cementation as low particle size distribution is a necessary feature for luting cements. The quicker set reaction of 'fast-set' highly viscous glass-ionomer cements may result in superior mechanical properties and good wear resistance as the material can theoretically achieve sufficient strength to resist masticatory loads within a shorter time. Hardness, strength (compressive and diametral tensile) and wear resistance of a 'fast-set' highly viscous GIC (Fuji IXGPfast) was examined. Its 'regular set' counterpart (Fuji IXGP) was used for comparison. It was found that besides being harder, the fast-set highly viscous $\mathrm{GIC}$ restorative offered nil other advantage in terms of physical property over the regular set counterpart thus implying that both are equally suited for restorative practice. As such the author has used these interchangeably (Personal Perspective).

Glass ionomers are not as strong in dynamic force application as compared to composite resin, from ex vivo study [38]. Investigation has been made into actual clinical performance, that being the final deciding 'measure' [39]. In this work, the performance of high-viscosity glass-ionomer cement (Fuji IXGP) was assessed in class-II restorations over a period of six years. The study group consisted of 116 class-II restorations in 72 patients. Distribution of restorations was: 30 mesioocclusal, 40 disto-occlusal, 46 mesio-occlusal-distal. Up to 18 months, nil failures were observed. From 18 to 42 months, restoration survival dropped to $93 \%$. At 72 months survival was only $60 \%$. The reason for replacement/repair was that of significant loss of Fuji IXGP in proximal areas. Restorations did not fail due to occlusal wear nor isthmus-fractures. Loss of GIC material in proximal areas, just below the contact point, was seen frequently. Clearly, Fuji IXGP performed less than ideally for interproximal situations over extended time frames and this is a feature of the restorative approach with these materials that must be borne in mind for clinical use. Perhaps surfacing the Fuji IXGP restoration in the contact areas with harder composite would provide a workable solution to this dilemma. Other considerations are discussed (below).
It has been indicated that in restorations avoiding proximal contacts, Fuji IXGP performs well in the atraumatic restorative treatment (ART) approach for posterior teeth in school children [40]. Clinical performance of Fuji IXGP over a year was completely satisfactory in Class I cavities. Another study showed that survival rates of class I ART restorations for Fuji IXGP in both primary and permanent teeth were high over a two year period in another Chinese investigation [41].

In order to counter any inherent strength weaknesses of Fuji IXGP several propositions have been brought into the foreground. Hydroxyapatite additive has been shown to enhance the flexural strength and fluoride ion release properties of viscous GIC such as Fuji IXGP [42]. Ultrasonic excitatory waves have been shown to increase early strength during this phase of the setting reaction and relates to temperature of the material [43]. Hence, ultrasonic may be a useful 'command-set' protocol for Fuji IXGP-enhancing compressive strength. As discussed above, ultrasonic waves enhance microtensile bond strength of high-viscosity GIC [29]. Additionally, and no doubt related to these observations, is that ultrasonic applied to Fuji IXGP during early setting notably decreases inner porosity of the material [44]. Reductions in such internal porosity with this material was $\sim 2.8-3.9 \%$. This would prevent crack propagation and material failure and hence increase apparent strength from early set.

Command setting through temperature induction has proved to be viable in terms of increasing early set strength of GIC [45]. Compressive strengths in the longer term were unaffected however. Curing temperatures of $60-70 \mathrm{C}$ were found to relate to these physical changes. The practicality of using such temperatures for command-set in clinical application remains to be determined and effects on surrounding soft tissue and pulp needs to be detailed.

As indicated above [31], by simply allowing time for material maturation, physical strength as measured through flexural capacity, has been shown to significantly improve. In other words, the operator on using Fuji IXGP would do well to inform their patients to avoid the restoration for at least $24 \mathrm{hr}$. It may be advised to lay out such instructions clearly in post-operative printed material to patients.

In terms of restoration strength, certain modifying conditions could be said to be present, such as water/saliva contamination at time of placement [46]. This study examined the effect of early water exposure on the shear strength of a spectrum of glass ionomer restoratives [46]. High strength, auto-cured Fuji IXGP, contrary to current teaching, was resistant to the mal-effects of early exposure to water. It was found that strength of auto-cure GIC in fact appeared to increase over a one year time frame. It would in fact appear that as the hydration of the GIC material increases on time, so does strength. Resin coating GIC on placement may well in fact interfere with this process of hydration and interfere with strength increase within the mouth. The performance of Fuji IXGP may therefore be improved by allowing water 
John A. Loudon, Oral Biology and Dentistry 2014,

exposure through not protecting the cement with a resin coating. It is certainly interesting that early water exposure did not detrimentally influence strength of glass ionomer. This is apparently contrary to instruction issued by most manufacturers and, indeed, teaching institutions. In summary, this argues contrary to the placement of resin coating over highstrength auto-cured GICs. The balanced argument suggests though that surface protection by resin prevents wear and increases flexural strength potentially by sealing voids/crazing formation which may be foci for material crack propagation (below).

The factor of wear resistance, of course, is of paramount practical consideration in restorative dentistry. Fuji IXGP has been compared to composites in this respect [47]. This study was performed ex vivo and it was noted that Fuji IXGP has comparable wear resistance to composite. It was summarized that Fuji IXGP may serve as a practical substitute for composites in low mechanical force situations where fluoride release is desirable and aesthetic requirements are not demonstrably high. Overall, this represents a 'tick' for general use-the limiting caveat being for particularly demanding cosmetic situations where surfacing with a fine layer of composite may be preferred.

A recent clinical study compared performance of Fuji IXGP to a resin composite [48]. First permanent molars of ninety one 11-12-year-old children (1-4 restorations per child) were restored with either Fuji IXGP or composite. At 3 years, $37 \%$ of Fuji IXGP restorations showed wear that could be graded as being slightly more than that of adjacent enamel and that of composite. This did not reach the level of statistical significance. Cosmetically, colour matching with the tooth of the $\mathrm{GIC}$ restorations improved over the period of the study. Only a small number of restorations failed due to secondary caries at three years. Thus, Fuji IXGP demonstrates acceptable wear for occlusal cavities in children with restoration of moderatedepth occlusal cavities in the first permanent molars in 11-12 year olds for up to three years.

In spite of the above, in order to address the criterion of surface wear various adaptations have been indicated. A nano-filled resin coating ( $\mathrm{G}$-coat Plus) has been used to reduce wear on GIC [48]. In this study, a trend was observed in relation to protection of Fuji IXGP against wear by G-coat Plus. Resin coating is perhaps quite a useful clinical support for GIC restorations. In this regard, coating offers coverage and resolution of notable surface defects such as crazing and voids, thus hindering crack initiation/spread and improving flexural strength [49]. In summary, nano-filled resin has been used to coat Fuji IXGP and significantly increased flexural strength and improved on general wear [50].

An important consideration in the dental clinic are 'iatrogenic' (dentist-related) causes for restoration problems. In this respect, certain topical fluoride formulations such as APF gel may damage the surface layer of restoratives such as Fuji IXGP [51]. In contrast, neutral sodium fluoride $2 \%$ gel did not deleteriously affect the surface of Fuji IXGP [51]. As such, a rational approach to managing this issue is for the clinician to be advised in avoiding APF formulations. The author would also suggest that it may prove to be of use to apply a nanofilled surface coating resin on Fuji IXGP restorations prior to fluoride application should the operator wish to continue their use of APF.

Another iatrogenic consideration concerns dental prophylaxis procedures, commonly used in day to day clinical dentistry. In regards Fuji IXGP, use of a rotating rubber cup with prophylaxis paste showed the most significant surface roughness [52]. The management of this scenario would be to simply avoid such a prophylaxis routine, or, to re-polish the GIC restorations after prophylaxis [52]. Surface texture irregularities are contra-indicated on restorative work as these may form foci for crack propagation/failure under repetitive loading as well as allow niches to form for dental plaque build-up.

As mentioned above [39], Fuji IXGP was notably seen to wear significantly in interproximal areas, thus leading to restoration demise. Coating the surface of interproximal restorations is practically not straightforward unless one has access by means of a restorative preparation in the adjacent tooth. Early hydration may increase Fuji IXGP strength [46]. In this respect, research may be guided to explore the significance of this for interproximal restorations. The author would recommend using command-set modalities such as ultrasonic (above) with encapsulated Fuji IXGP to aim to assist in resolving the dilemma in respect to at least early onset proximal GIC wear. Post-operative instructions in writing advising care with placing force in the area of the restoration within the first $24 \mathrm{hr}$ is also advised. Having said that, the author considers this particular area of weakness of GIC requires significant added investigation.

Apart from coronal restoration, repair of root caries lesions is becoming a prime feature in dentistry at the present time. This is due in large part to patients keeping their teeth for longer and the prevalence of gingival recession secondary to periodontal disease. Root caries repair lends itself particularly well to a minimally invasive approach. Minimal invasion dentistry is relevant in this context as there is a high incidence with $>\sim 20 \%$ prevalence root caries in elderly people in areas where there has been recession of the gingivae and exposed root surface [53]. Glass ionomer is a restorative material that seems particularly well suited for restoring root lesions as it has been stated the material has good aesthetic and anticaries abilities [53]. Cosmetic properties and direct loading strength of the material is of less importance in this scenario as compared to bonding capacity to dentine and fluoride release. In these latter respects, glass ionomer cements are ideally suited particularly in the light of the fact that moisture contamination plays a less significant role for auto-cured glass ionomers such as Fuji IXGP (above). GIC restorations survived adequately well under the rigorous environment of the root 
John A. Loudon, Oral Biology and Dentistry 2014,

surface [54]. Restorations showed a cumulative survival rate of $\sim 77 \%$ at 80 months. Periodontal status was unrelated to restoration survival.

One very important characteristic for any restorative material is that it is radio-opaque. This is important as the clinician should be able to distinguish the restorative material or cement from any adjacent decalcified tooth structure [55]. From an investigation into this with regards Fuji IXGP, it has been concluded that this material demonstrates sufficient radio-opacity for diagnostic scenarios.

The literature places concerns forward regarding the intrinsic strength of Fuji IXGP for core buildup scenarios [56]. By in vitro analysis, fatigue resistance of two glass ionomer cements, viz: Shofu Hi-Dense and Fuji IXGP were compared for their mechanical properties as core materials under loading versus silver-reinforced glass ionomer (Ketac-Silver) and amalgam [56]. Fuji IXGP when used as core material showed higher number of defects than amalgam. These results were consistent with the notion that its fatigue resistance may be not as durable as compared to amalgam. It may still remain true to say that Fuji IXGP is still suited for core buildup protocols but that it does not match a 'gold standard', viz: amalgam. This does not necessarily mean that this material is unsuited for that purpose (Personal perspective).

In dealing with core strength it may be practical for the operator to apply various 'command-set' protocols (above) to improve on the pbysical properties. The operator also ought to make certain the surface of the prepared core is polished to a high level of smoothness so as to avoid surface irregularities that may result in focal physical stress build-up leading to crack initiation. Future developments incorporating hydroxyapatite particles into Fuji IXGP may well also aid in solving the dilemma in respect to physical core weakness [42]

In terms of cementation, there is a dearth of information on the use of new generational GICs such as Fuji IXGP. One reason for this is that the material is viscous and concerns could be raised against its adaptation to the fitting surface of the crown or post. Clearly, well defined studies need to be performed to examine this under controlled clinical conditions. Use of the GIC cement Fuji Cap 1, an encapsulated cement, has been examined along with zinc phosphate cement ex vivo for cementing silver-palladium crowns at varying seating forces and with varying marginal designs (chamfer, shoulder and shoulder with $45^{\circ}$ bevel) [11]. Higher seating forces produced improved crown seating although did not affect retention. Shoulder and shoulder with bevel finish impacted positively on crown retention compared to chamfer. Cementation with GIC provided for improved retention over zinc phosphate. In total, marginal seal is seen to be influenced by seating force-the greater the force the greater the seal [11]. Crown retention is influenced by marginal finish type and the actual lute cement. The message here for clinicians considering use of Fuji IXGP therefore is to employ positive seating forces and preferably enable crowns to have some form of shoulder preparation on as many aspects of the preparation as possible. Attention to details such as anti-rotational features and degree of taper also ought to be borne in mind. Future studies shall be required to investigate in detail the comparative qualities of Fuji IXGP as a crown luting agent under controlled laboratory conditions. Having said this, the author considers that Fuji IXGP would not be suited as a lute agent for porcelain laminate veneers-the material is too viscous for that specific application which in itself is a rather specialized technique in its own right within the field of dental cementation and calls on cements with particular and unique properties (for example, cosmetics).

In terms of post cementation, bond strength to radicular dentine needs to be adequate. In this respect, controlled experimental studies have yet to be carried out to examine whether Fuji IXGP performs adequately to shear, torsion, compression and tensional forces in that scenario. A key matter, no matter what cementation is used, relates to other features such as post-length and occlusal force distribution and height-length ratio of post to restoration. These other considerations in terms of biomechanical dentistry outweigh reliance on a particular cementation system. This is in fact a principle to be considered with all the above applications for Fuji IXGP. Biomechanical features, with particular emphasis on occlusion, have a very significant, and at times, over-riding influence and need to be seriously taken into account. These factors in toto may well outbalance the intrinsic mechanical characteristics of any given restorative/cement. It is notable that cement film thickness along with post size have significant bearings on pullout strength of fiber-reinforced posts [57]. Highest bonding strengths were not obtained with the thinnest cement layer but with larger post sizes. Over a certain cement thickness however the retentive bond was compromised thus interfering with resistance to dislocation of the post [57]. Post retention is related to a number of variables such as the endodontic treatment, root canal morphology, post space preparation, post type and lute cement itself which all may impact on effective outcome [58]. Various pretreatment approaches for the post surface such as silicoating/silanization of fiber-reinforced composite posts are also considered relevant [58]. Preservation of tooth tissue with presence of a ferrule certainly is to be considered in respect to long term post retention [58] in addition to adhesive chemistry. Overall, future research would be advised to focus on the effectiveness and optimization for employing high viscosity GICs for post cementation. In general terms though the key message in respect to post retention is that the clinician cannot unduly lean on a material's properties to make up for deficiencies in exacting technique or diagnosis.

Glass ionomer cements lend themselves rather well for use as temporary restorations-even when there has been considerable loss of coronal tooth structure. Ample fluoride release, suitable bonding to underlying tooth structure and adequate physical properties have made these popular in the ART protocol and for endodontic applications. The material 
John A. Loudon, Oral Biology and Dentistry 2014,

is useful in this situation for extended periods of a number of months. It proves physically adequate and minimizes the 'all important' aspect of coronal leakage which is strongly detrimental and may lead to contamination of the canals being prepared. In fact, in terms of sealing against Streptococcus mutans bacterial contamination, GICs perform better than zinc oxide eugenol [59]. Possibly this is due to the intrinsic ability of the cement to chemically bond to underlying tooth structure as well as release fluoride ions which may have bacterio-static properties.

Despite the many instances where Fuji IXGP is suited for dental use, there may be circumstances where this material is not clinically recommended, as supported by evidence. For example, as a bracket cementing agent in orthodontics [60]. Perhaps by employing a number of techniques aimed to improve the physical properties of Fuji IXGP as discussed (above) may well aim to solve these limitations. Research requires to be performed to address such points.

\section{Conclusion}

For each dental clinical application I have outlined the benefits and downsides for Fuji IXGP and have provided suggestion on how to overcome limitations in each situation. Key 'take home messages' consider the fact that dental materials do not overcome deficiencies in diagnosis nor technique and that ongoing research is needed to develop GIC further for dentistry. The search for the 'all purpose' material is idealistic and challenging yet relies heavily on clinician ability to a large extent. In this respect, the dentist must be adaptive and ever-resourceful in practice-necessitating the clinician to combine technique and application in the best possible fashion yet be open to developments in the field. This calls upon dentists as clinical scholars and to be innovative and investigative in practice thus striving towards improved understandings that translate into practical and workable solutions for our patients.

By keeping an open mind and flexible in our options and approach we may well find that ultimate material for dental science. It is my intent to consider Fuji IXGP as a significant step towards that goal. In a manner of speaking the topic is not yet set in cement-literally - but future efforts by dental practitioners may aid in bringing this into a more 'concrete' reality.

\section{Competing interests}

The author declares that he has no competing interests.

\section{Acknowledgement}

I thank Dr George Al-Horani, staff and dental patients of Wetherill Park Medical Centre who have been my support during the preparation of this article. I also thank the reviewers for their time and useful suggestions.

\section{Publication history}

Editor: Cássio do Nascimento, University of São Paulo, Brazil. EIC: Thimios A. Mitsiadis, University of Zurich, Switzerland. Received: 03-Feb-2014 Final Revised: 17-Mar-2014 Accepted: 14-Apr-2014 Published: 24-Apr-2014

\section{References}

1. Wilson $A D$ and Kent $B E$. A new translucent cement for dentistry. The glass ionomer cement. Br Dent J. 1972; 132:133-5. | Article | PubMed

2. Zilberman $U$. Ion exchanges between glass-ionomer restorative material and primary teeth components-an in vivo study. Oral Biol Dent. 2014; 2:1. I Pdf

3. Bresciani E, Barata Tde J, Fagundes TC, Adachi A, Terrin MM and Navarro MF. Compressive and diametral tensile strength of glass ionomer cements. J Appl Oral Sci. 2004; 12:344-8. I Article I PubMed

4. Mickenautsch $S$ and Yengopal V. Failure rate of high-viscosity GIC based ART compared with that of conventional amalgam restorations-evidence from an update of a systematic review. SADJ. 2012; 67:329-31. | Article | PubMed

5. Yap AU, Pek YS and Cheang P. Physico-mechanical properties of a fastset highly viscous GIC restorative. J Oral Rehabil. 2003; 30:1-8. | Article I PubMed

6. Banomyong D, Palamara JE, Burrow MF and Messer HH. Effect of dentin conditioning on dentin permeability and micro-shear bond strength. Eur J Oral Sci. 2007; 115:502-9. | Article | PubMed

7. Mickenautsch S, Yengopal V, Leal SC, Oliveira LB, Bezerra AC and Bonecker M. Absence of carious lesions at margins of glass-ionomer and amalgam restorations: a meta- analysis. Eur J Paediatr Dent. 2009; 10:41-6. | Pdf | PubMed

8. Yip HK, Tay FR, Ngo HC, Smales RJ and Pashley DH. Bonding of contemporary glass ionomer cements to dentin. Dent Mater. 2001; 17:456-70. | Article | PubMed

9. Suwatviroj P, Messer LB and Palamara JE. Microtensile bond strength of tooth-colored materials to primary tooth dentin. Pediatr Dent. 2004; 26:67-74. | Article | PubMed

10. Li ZC and White SN. Mechanical properties of dental luting cements. J Prosthet Dent. 1999; 81:597-609. | Article | PubMed

11. Piemjai M. Effect of seating force, margin design, and cement on marginal seal and retention of complete metal crowns. Int $J$ Prosthodont. 2001; 14:412-6. | PubMed

12. Bonfante G, Kaizer OB, Pegoraro LF and do Valle AL. Tensile bond strength of glass fiber posts luted with different cements. Braz Oral Res. 2007; 21:159-64. | Article | PubMed

13. Rodig T, Nusime AK, Konietschke F and Attin T. Effects of different luting agents on bond strengths of fiber-reinforced composite posts to root canal dentin. J Adhes Dent. 2010; 12:197-205. | Article | PubMed

14. Putignano A, Poderi G, Cerutti A, Cury A, Monticelli F, Goracci $C$ and Ferrari M. An in vitro study on the adhesion of quartz fiber posts to radicular dentin. J Adhes Dent. 2007; 9:463-7. | PubMed

15. Sailer I, Pjetursson BE, Zwahlen M and Hammerle CH. A systematic review of the survival and complication rates of all-ceramic and metalceramic reconstructions after an observation period of at least 3 years. Part II: Fixed dental prostheses. Clin Oral Implants Res. 2007; 18 Suppl 3:86-96. | Article | PubMed

16. Kopp FR, Belser UC and Hess D. Invisible supragingival crown margins: an in vitro study. Int J Prosthodont. 1989; 2:331-41. | PubMed

17. Wiegand $A$, Schmid $M$ and Schmidlin PR. [Esthetic repair of crown margins with composite. A case report]. Schweiz Monatsschr Zahnmed. 2008; 118:427-35. | PubMed

18. Smales RJ and Gao W. In vitro caries inhibition at the enamel margins of glass ionomer restoratives developed for the ART approach. J Dent. 2000; 28:249-56. | Article | PubMed

19. Francci C, Deaton TG, Arnold RR, Swift EJ, Jr., Perdigao J and Bawden JW. Fluoride release from restorative materials and its effects on dentin mineralization. J Dent Res. 1999; 78:1647-54. | Article | PubMed

20. Jang KT, Garcia-Godoy F, Donly KJ and Segura A. Remineralizing effects of glass ionomer restorations on adjacent interproximal caries. ASDC $J$ Dent Child. 2001; 68:125-8, 142. | PubMed 
John A. Loudon, Oral Biology and Dentistry 2014,

21. Ngo HC, Mount G, Mc Intyre J, Tuisuva J and Von Doussa RJ. Chemical exchange between glass-ionomer restorations and residual carious dentine in permanent molars: an in vivo study. J Dent. 2006; 34:608-13. | Article | PubMed

22. Hu JY, Li YQ, Smales RJ and Yip KH. Restoration of teeth with moreviscous glass ionomer cements following radiation-induced caries. Int Dent J. 2002; 52:445-8. | Article | PubMed

23. Czarnecka B, Deregowska-Nosowicz P, Limanowska-Shaw $\mathrm{H}$ and Nicholson JW. Shear bond strengths of glass-ionomer cements to sound and to prepared carious dentine. J Mater Sci Mater Med. 2007; 18:8459. | Article | PubMed

24. Tanumiharja M, Burrow MF and Tyas MJ. Microtensile bond strengths of glass ionomer (polyalkenoate) cements to dentine using four conditioners. J Dent. 2000; 28:361-6. | Article | PubMed

25. Kulczyk KE, Sidhu SK and McCabe JF. Salivary contamination and bond strength of glass-ionomers to dentin. Oper Dent. 2005; 30:676-83. | PubMed

26. Rekha CV, Varma B and Jayanthi. Comparative evaluation of tensile bond strength and microleakage of conventional glass ionomer cement, resin modified glass ionomer cement and compomer: An in vitro study. Contemp Clin Dent. 2012; 3:282-7. I Article I PubMed Abstract | PubMed Full Text

27. Lopes JF, Vergani CE, Giampaolo ET, Pavarina AC and Machado AL. Shear bond strength fatigue limit of rest seats made with dental restoratives. J Adhes Dent. 2007; 9:203-8. | PubMed

28. Gorseta K, Skrinjaric T and Glavina D. The effect of heating and ultrasound on the shear bond strength of glass ionomer cement. Coll Antropol. 2012; 36:1307-12. | Article | PubMed

29. Azevedo ER, Coldebella CR and Zuanon AC. Effect of ultrasonic excitation on the microtensile bond strength of glass ionomer cements to dentin after different water storage times. Ultrasound Med Biol. 2011; 37:21338. | Article | PubMed

30. Zhang Y, Burrow MF, Palamara JE and Thomas CD. Bonding to glass ionomer cements using resin-based adhesives. Oper Dent. 2011; 36:618 25. | Article | PubMed

31. Irie M, Maruo Y, Nishigawa G, Suzuki K and Watts DC. Class I gapformation in highly-viscous glass-ionomer restorations: delayed vs immediate polishing. Oper Dent. 2008; 33:196-202. | Article | PubMed

32. Singla T, Pandit IK, Srivastava N, Gugnani N and Gupta M. An evaluation of microleakage of various glass ionomer based restorative materials in deciduous and permanent teeth: An in vitro study. Saudi Dent J. 2012; 24:35-42. | Article | PubMed Abstract | PubMed Full Text

33. Gorseta K, Glavina D and Skrinjaric I. Influence of ultrasonic excitation and heat application on the microleakage of glass ionomer cements. Aust Dent J. 2012; 57:453-7. | Article | PubMed

34. Ferreira Fde M, do Vale MP, Jansen WC, Paiva SM and Pordeus IA. Effect of mixing process on microleakage of glass ionomer cements used in atraumatic restorative treatment on primary molars. J Clin Pediatr Dent. 2007; 31:251-6. | Article | PubMed

35. Masih S, Thomas AM, Koshy G and Joshi JL. Comparative evaluation of the microleakage of two modified glass ionomer cements on primary molars. An in vivo study. J Indian Soc Pedod Prev Dent. 2011; 29:135-9. Article | PubMed

36. Castro A and Feigal RE. Microleakage of a new improved glass ionomer restorative material in primary and permanent teeth. Pediatr Dent. 2002; 24:23-8. | Pdf | PubMed

37. Piemjai M, Watanabe A, Iwasaki Y and Nakabayashi N. Effect of remaining demineralised dentine on dental microleakage accessed by a dye penetration: how to inhibit microleakage? J Dent. 2004; 32:495501. | Article | PubMed

38. Ji W, Chen Z and Frencken JE. Strength of tunnel-restored teeth with different materials and marginal ridge height. Dent Mater. 2009; 25:1363-70. | Article | PubMed

39. Scholtanus JD and Huysmans MC. Clinical failure of class-II restorations of a highly viscous glass-ionomer material over a 6-year period: a retrospective study. J Dent. 2007; 35:156-62. I Article | PubMed

40. Luo Y, Wei SH, Fan MW and Lo EC. Clinical investigation of a highstrength glass ionomer restorative used with the ART technique in Wuhan, China: one-year results. Chin J Dent Res. 1999; 2:73-8. | Article | PubMed

41. Lo EC, Luo Y, Fan MW and Wei SH. Clinical investigation of two glassionomer restoratives used with the atraumatic restorative treatment approach in China: two-years results. Caries Res. 2001; 35:458-63. I Article I PubMed

42. Arita K, Yamamoto A, Shinonaga Y, Harada K, Abe Y, Nakagawa K and Sugiyama $S$. Hydroxyapatite particle characteristics influence the enhancement of the mechanical and chemical properties of conventional restorative glass ionomer cement. Dent Mater J. 2011; 30:672-83. | Article | PubMed

43. Kleverlaan CJ, van Duinen RN and Feilzer AJ. Mechanical properties of glass ionomer cements affected by curing methods. Dent Mater. 2004; 20:45-50. | Article | PubMed

44. Coldebella CR, Santos-Pinto $L$ and Zuanon AC. Effect of ultrasonic excitation on the porosity of glass ionomer cement: a scanning electron microscope evaluation. Microsc Res Tech. 2011; 74:54-7. | Article | PubMed

45. Algera TJ, Kleverlaan CJ, Prahl-Andersen B and Feilzer AJ. The influence of environmental conditions on the material properties of setting glassionomer cements. Dent Mater. 2006; 22:852-6. | Article | PubMed

46. Wang XY, Yap AU and Ngo HC. Effect of early water exposure on the strength of glass ionomer restoratives. Oper Dent. 2006; 31:584-9. | Article | PubMed

47. Yap AU, Teo JC and Teoh SH. Comparative wear resistance of reinforced glass ionomer restorative materials. Oper Dent. 2001; 26:343-8. | PubMed

48. Diem VT, Tyas MJ, Ngo HC, Phuong LH and Khanh ND. The effect of a nano-filled resin coating on the 3-year clinical performance of a conventional high-viscosity glass-ionomer cement. Clin Oral Investig. 2014; 18:753-9. | Article | PubMed

49. Zoergiebel J and Ilie N. Evaluation of a conventional glass ionomer cement with new zinc formulation: effect of coating, aging and storage agents. Clin Oral Investig. 2013; 17:619-26. | Article | PubMed

50. Bonifacio CC, Werner A and Kleverlaan CJ. Coating glass-ionomer cements with a nanofilled resin. Acta Odontol Scand. 2012; 70:471-7. | Article | PubMed

51. Ozdemir-Ozenen D, Sungurtekin E, Issever H and Sandalli N. Surface roughness of fluoride-releasing restorative materials after topical fluoride application. Eur J Paediatr Dent. 2013; 14:68-72. | Article | PubMed

52. Wu SS, Yap AU, Chelvan S and Tan ES. Effect of prophylaxis regimens on surface roughness of glass ionomer cements. Oper Dent. 2005; 30:180 4. | Article | PubMed

53. Teich S and Gilboa I. A minimally invasive restorative approach for treatment of interproximal root caries lesions. Quintessence Int. 2011; 42:611-4. I PubMed

54. Gilboa I, Cardash HS, Baharav H, Demko CA and Teich ST. A longitudinal study of the survival of interproximal root caries lesions restored with glass ionomer cement via a minimally invasive approach. Gen Dent. 2012; 60:e224-30. | PubMed

55. DuBois DJ, Reichl RB and Hondrum SO. The comparative radiopacity of Fuji IX-GP, an intermediate restorative material. Mil Med. 2000; 165:278-82. | PubMed

56. Gateau P, Sabek M and Dailey B. In vitro fatigue resistance of glass ionomer cements used in post-and-core applications. J Prosthet Dent. 2001; 86:149-55. I Article | PubMed

57. D'Arcangelo $C$, Cinelli $M$, De Angelis $F$ and D'Amario $M$. The effect of resin cement film thickness on the pullout strength of a fiber-reinforced post system. J Prosthet Dent. 2007; 98:193-8. | Article | PubMed 
John A. Loudon, Oral Biology and Dentistry 2014,

http://www.hoajonline.com/journals/pdf/2053-5775-2-5.pdf

58. Goracci $C$ and Ferrari M. Current perspectives on post systems: a literature review. Aust Dent J. 2011; 56 Suppl 1:77-83. | Article | PubMed

59. Seiler KB. An evaluation of glass ionomer-based restorative materials as temporary restorations in endodontics. Gen Dent. 2006; 54:33-6. I PubMed

60. Algera TJ, Kleverlaan CJ, Prahl-Andersen B and Feilzer AJ. The influence of different bracket base surfaces on tensile and shear bond strength. Eur $J$ Orthod. 2008; 30:490-4. | Article | PubMed

\section{Citation:}

Loudon JA. Next generational fuji IX-a proposed universal dental material-but not yet 'set in cement'. Oral Biol Dent. 2014; 2:5.

http://dx.doi.org/10.7243/2053-5775-2-5 